\title{
Degradabilidade dos agentes quelantes EDTA e EDDS após aplicação no solo
}

\author{
Antonieta Aparecida Fiori ( ${ }^{()}$; Mônica Ferreira de Abreu $\left(2^{*}\right)$; Aline Renée Coscione $\left({ }^{2}\right)$, Cristiano \\ Alberto de Andrade $\left({ }^{2}\right)$; Cleide Aparecida de Abreu ( ${ }^{2}$ ) \\ (') Instituto Agronômico (IAC), Programa de Pós-graduação em Agricultura Tropical e Subtropical, Caixa Postal 28, $13012-970$ \\ Campinas (SP). \\ (2) IAC, Centro de Pesquisa e Desenvolvimento de Solos e Recursos Ambientais, Caixa Postal 28, 13012-970 Campinas (SP). \\ (*) Autora correspondente: monica@iac.sp.gov.br
}

Recebido: 9/jun./2010; Aceito: 23/nov./2010.

\begin{abstract}
Resumo
Este trabalho objetivou avaliar a degradabilidade dos quelantes EDTA e EDDS por meio do monitoramento da respiração do solo. Os quelantes foram aplicados em dois solos, um Cambissolo Háplico contaminado por metais pesados e um Latossolo Vermelho-Amarelo não contaminado. Três doses de cada quelante (250, 500 e $\left.750 \mathrm{mg} \mathrm{kg}^{-1}\right)$ e uma dose de glicose foram aplicadas em ambos os solos testados, com três repetições cada uma. A degradação dos quelantes foi determinada por meio da quantificação do $\mathrm{CO}_{2}$ emanado do início até o término do ensaio. As equações de cinética química não se ajustaram satisfatoriamente para ambos os quelantes no Latossolo, enquanto para o Cambissolo Háplico resultaram em valores de meia-vida entre 12-16 dias para o EDDS e de 6 dias para o EDTA. Nesse período, a quantidade degradada de EDTA foi de 15\% somente. Para o Latossolo o EDTA foi responsável pela menor taxa de degradação, próxima de zero, ocorrida na menor dose, e o EDDS pela maior taxa (135\%), ocorrida na maior dose. Para o Cambissolo Háplico, a menor taxa de degradação $(19,2 \%)$ coube ao EDTA na maior dose e a maior taxa (211,4\%), ao EDDS na menor dose. Ambos os quelantes tiveram redução da taxa de degradação com o aumento de suas respectivas doses, exceto com a aplicação da menor dose do EDDS (250 $\mathrm{mg} \mathrm{kg}^{-1}$ ). Contudo, a degradabilidade do EDDS continuou maior que a verificada com a aplicação do EDTA.
\end{abstract}

Palavras-chave: degradabilidade, cinética química, carbono degradado, EDTA.

\section{Degradability of chelant agent EDTA and EDDS after application on soil}

\section{Abstract}

This work aimed to evaluate the biodegradation of the chelant agents EDDS and EDTA through soil respiration. The chelant agents were applied in two soils, one was an Inceptisol contaminated with heavy metals and the other was a no contaminate Oxisol. Three doses of each chelant (250, 500 and $\left.750 \mathrm{mg} \mathrm{kg}^{-1}\right)$ and one dose of glucose were applied in both the soils tested, with three replicates. The chelant biodegradation was determined by quantifying the $\mathrm{CO}_{2}$ emitted during the experiment. The chemical kinetics equations used did not fit the data satisfactory for both chelants on the Oxisol, while a half-life of $12-16$ days for EDDS and of 6 days for EDTA was obtained for the Inceptisol. During the half-life period, a chelant degradation of only $15 \%$ was observed. For the Oxisol, EDTA was responsible for the lowest biodegradation rate, next to zero, which occurred at lowest dose added. EDDS caused the highest biodegradation (135\%) at the highest dose added to the soil. For the Inceptisol, the lowest biodegradation rate (19.2\%) was observed with the highest dose of EDTA and the largest degradation rate (211.4\%) with the EDDS at the lowest dose. Both the chelants degraded less with the increase of their respective doses, except with the application of the lowest dose of the EDDS $\left(250 \mathrm{mg} \mathrm{kg}^{-1}\right)$. However, the biodegradation of the EDDS continued greater than that verified with the EDTA application.

Key words: Biodegradation, chemical kinetics, degraded carbon, EDTA. 


\section{INTRODUÇÃO}

O tratamento de áreas contaminadas ou poluídas é usualmente conhecido como remediação e pode envolver medidas para eliminar, imobilizar ou reduzir o contaminante para níveis aceitáveis e previamente definidos. Um dos métodos empregados envolve a adição de quelantes, cujo papel é evitar a precipitação e/ou adsorção dos metais pesados na fase sólida do solo, podendo ser empregados agentes inorgânicos e orgânicos, como o EDTA (etileno diaminotetraacético).

O problema é que os complexos metais-quelantes podem ser lixiviados no perfil do solo, representando um risco real de contaminação das águas subterrâneas (MADRID et al. 2003; LASAT, 2002). Esse efeito é potencializado não apenas quando doses elevadas do quelante são aplicadas, mas também quando este permanece ativo no solo por período relativamente longo. Dentre os quelantes sintéticos, o EDTA é o mais estudado e difundido comercialmente, mas sua extensa persistência no ambiente, até 15 anos, o torna alvo de severas críticas por parte dos ambientalistas. Outro agente quelante, o EDDS (etileno diaminodisuccínio), vem sendo amplamente utilizado pela indústria química, principalmente por ser relativamente mais biodegradável, tornando-o componente preferencial a outros agentes quelantes mais persistentes na formulaçáo de detergentes (JaWORSKA et al., 1999). Aliado à degradabilidade, seu desempenho na remoção de metais pesados, o torna um potencial substituto ao agente quelante comumente utilizado, EDTA (etileno diaminotetraacético).

Por outro lado, não se pode ignorar que quanto mais facilmente degradado for o quelante no solo, provavelmente mais curto será seu período de ação, o que pode conduzir a resultados insatisfatórios quanto à remediaçấo de áreas contaminadas. Desse modo, as informaçôes sobre o processo de degradação dos agentes quelantes após aplicação no solo são importantes para o uso eficiente e ambientalmente responsável da referida técnica de remediação.

Conforme exposto, o principal diferencial entre os agentes quelantes EDTA e EDDS é o tempo de degradabilidade, de modo que o objetivo deste trabalho foi o de determinar e comparar o comportamento da degradabilidade dos quelantes em diferentes solos. Os referidos quelantes foram aplicados em um Cambissolo Háplico distrófico textura argilosa (EMBRAPA, 1999) com altos teores de metais pesados (contaminado) e em Latossolo Vermelho-Amarelo distrófico típico textura argilosa (EMBRAPA, 1999), sem contaminação por metais pesados.

\section{MATERIAL E MÉTODOS}

Considerando que os agentes quelantes tem potencial de uso na remediação de solos contaminados com metais pesados e que a atividade microbiana edáfica pode ser afetada pelo nível e tipo de contaminação com essas espécies químicas, foram escolhidos dois solos (camada $0-20 \mathrm{~cm}$ ) para a avaliaçáo da degradabilidade - um solo denominado "não contaminado" e outro, "contaminado".

O solo não contaminado, classificado como Latossolo Vermelho-Amarelo distrófico típico, textura argilosa (ЕмBRAPA, 1999), foi coletado na Fazenda Santa Elisa, Campinas (SP). Alguns atributos químicos desse solo na camada superficial foram: $\mathrm{pH}_{\mathrm{H} 2 \mathrm{O}}=4,9$; carbono total $=13 \mathrm{~g} \mathrm{~kg}^{-1}$; soma de bases $(\mathrm{SB})=18 \mathrm{mmol} \mathrm{dm}^{-3}$; saturaçáo por bases $(\mathrm{V} \%)=28 \%$; saturação por alumínio $(\mathrm{m})=37 \%$; capacidade de troca catiônica $\left(\right.$ CTC) $=60 \mathrm{mmol} \mathrm{dm}^{-3}$; teor de argila $=370 \mathrm{~g} \mathrm{~kg}^{-1}$ (OliveIra et al., 2007).

O solo contaminado, Cambissolo háplico distrófico, textura argilosa (ЕмBrapa, 1999), foi coletado de gleba com cerca de 2,75 ha, onde foi adicionado resíduo de sucata automobilística. A área, cultivada com cana-de-açúcar, recebeu o resíduo com uma incorporação via gradagem e aplicação de calcário dolomítico $\left(10\right.$ tha $\left.^{-1}\right)$ visando estabilizar os metais no solo. A análise química deste solo, feita com base no sistema IAC de análise de solo (RAIJ et al., 2001), resultou nos seguintes resultados: $\mathrm{pH}_{\mathrm{CaCl} 12}=6,9, \mathrm{C}$ orgânico total $=73 \mathrm{~g} \mathrm{~kg}^{-1} ; \mathrm{SB}=257 \mathrm{mmol}_{\mathrm{c}} \mathrm{dm}^{-3} ; \mathrm{K}=2 \mathrm{mmol} \mathrm{dm}^{-3}$; $\mathrm{Ca}=213 \mathrm{mmol}_{\mathrm{c}} \mathrm{dm}^{-3} ; \quad \mathrm{Mg}=42 \mathrm{mmol}_{\mathrm{c}} \mathrm{dm}^{-3} ; \mathrm{V}=$ $96 \% ; \mathrm{m}=0 \%$; CTC $=267,8 ; \quad \mathrm{P}=25 \mathrm{mgdm}^{-3}$; teor de argila $=363 \mathrm{~g} \mathrm{~kg}^{-1}$; teor de silte $=399 \mathrm{~g} \mathrm{~kg}^{-1}$, teor de areia $=238 \mathrm{~g} \mathrm{~kg}^{-1} ; \mathrm{Cd}=5,9 \mathrm{mg} \mathrm{kg}^{-1}$ de massa seca, $\mathrm{Pb}=358,9$ $\mathrm{mg} \mathrm{kg}{ }^{-1}$ de massa seca; $\mathrm{Cu}=206,0 \mathrm{mg} \mathrm{kg}^{-1}$ de massa seca; $\mathrm{Cr}=58,4 \mathrm{mg} \mathrm{kg}^{-1}$ de massa seca; $\mathrm{Ni}=26,9 \mathrm{mg} \mathrm{kg}^{-1}$ de massa seca; $\mathrm{Zn}=1.495 \mathrm{mg} \mathrm{kg}^{-1}$ de massa seca; $\mathrm{Fe}=18.830 \mathrm{mg} \mathrm{kg}^{-1}$ de massa seca.

As amostras de solo foram secas ao ar, passadas em peneira de malha de $2 \mathrm{~mm}$ (TFSA), devidamente homogeneizadas e reservadas para a realização dos experimentos. Três subamostras de cada solo foram utilizadas para determinação da capacidade de retenção de água (CRAS), utilizada posteriormente para ajuste da umidade de incubação. Os valores de CRAS foram iguais a 285 e $313 \mathrm{~mL}$ $\mathrm{kg}^{-1}$, respectivamente, para os solos não contaminados e contaminados.

Os tratamentos foram montados com três repetiçóes e constituíram de dois quelantes, EDTA e EDDS, aplicados em três doses correspondentes a 250; 500 e 750 $\mathrm{mg} \mathrm{kg}{ }^{-1}$; uma dose de glicose $\left(400 \mathrm{mg} \mathrm{kg}^{-1}\right)$ equivalente à massa de carbono da dose intermediária dos quelantes; e um controle (somente solo). Os tratamentos foram aplicados nos dois solos, porém não foi objetivo compará-los, mas sim caracterizar o processo de degradação dos quelantes em ambos os casos. A aplicação dos quelantes e da glicose foi feita via soluçáa aquosa, descontada água no posterior ajuste da umidade para incubaçáo.

Antes de receber os tratamentos, os solos sofreram préincubação de sete dias com umidade ajustada a $50 \%$ da CRAS, visando ativar e uniformizar a atividade microbiana. A incubação foi realizada em frascos de vidro com diâmetro de $12 \mathrm{~cm}$ e capacidade para 1,3 L, com tampa plástica para 
fechamento hermético. Os frascos foram preenchidos com solo até a metade da sua altura, para que houvesse espaço suficiente para posterior acondicionamento do frasco plástico com $\mathrm{NaOH}$, que foi colocado sobre uma tela de nylon (12 $\mathrm{cm}$ de diâmetro) que o separava do contato com o solo.

O frasco de plástico com capacidade de 50 $\mathrm{mL}$ continha $40 \mathrm{~mL}$ de solução padronizada de $\mathrm{NaOH} 0,25 \mathrm{~mol} \mathrm{~L}^{-1}$, para reação com o $\mathrm{CO}_{2}$ liberado (Coscione e Andrade, 2006). Os frascos contendo o $\mathrm{NaOH}$ foram periodicamente trocados, isto é, as trocas ocorriam em função da degradação. No início do experimento as trocas eram diárias e com intervalos maiores no fim do experimento. A cada troca, os frascos eram substituídos por outros, sendo aqueles retirados do interior dos frascos de vidro, encaminhados imediatamente para quantificação do $\mathrm{CO}_{2}$ liberado. Os tratamentos foram incubados com umidade correspondente a $70 \%$ da CRAS, a $28 \pm 2{ }^{\circ} \mathrm{C}$ e no escuro, sendo o período de incubaçáo de 50 dias para o experimento com solo não contaminado e de 43 dias para o solo contaminado.

A quantificaçáo do carbono liberado na forma de $\mathrm{CO}_{2}\left(\mathrm{C}-\mathrm{CO}_{2}\right)$ nos tratamentos foi realizada por meio de leitura da condutividade elétrica da solução de $\mathrm{NaOH}$. O fundamento deste método baseia-se na redução da condutividade elétrica da solução de $\mathrm{NaOH}$, na medida em que há formação de carbonato, função da reação do $\mathrm{CO}_{2} \mathrm{com}$ o NaOH (Rodella e Saboya, 1999).

A quantidade de $\mathrm{C}-\mathrm{CO}_{2}$ liberada e atribuída à degradação de cada quelante ou glicose foi obtida descontandose de cada tratamento com adição de carbono (EDTA, EDDS e glicose) o valor médio de $\mathrm{C}-\mathrm{CO}_{2}$ liberado no respectivo controle.

Com os resultados de $\mathrm{C}-\mathrm{CO}_{2}$ acumulados foram feitos ajustes tentativos de modelo de cinética química de primeira ordem com uma fase, usando o programa CURVE EXPERT 1.3 (2007), para obtenção de alguns parâmetros de cinética química:

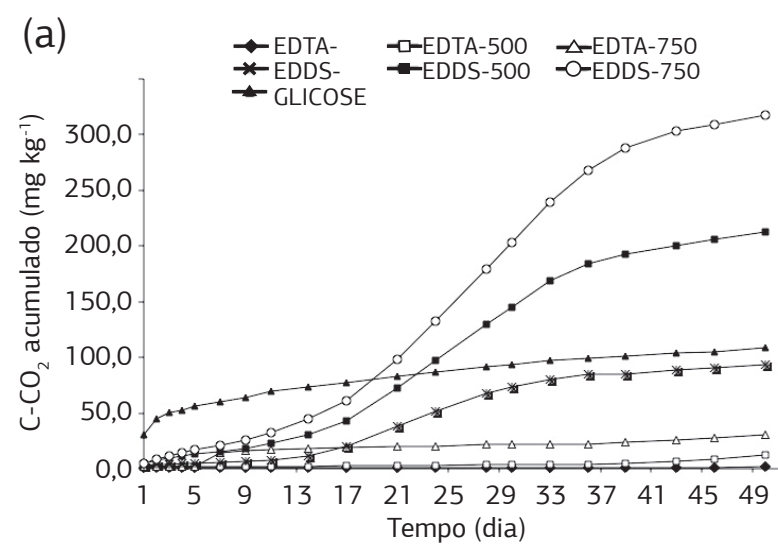

$\mathrm{C}_{\text {degradado }}=\mathrm{C}_{0} \cdot\left(1-\mathrm{e}^{-\mathrm{kt}}\right)$

Em que:

$\mathrm{C}_{\text {degradado }}=$ quantidade $\left(\mathrm{mg} \mathrm{kg}^{-1}\right)$ de carbono na forma de $\mathrm{CO}_{2}$ liberado no tempo t;

$\mathrm{C}_{0}=$ quantidade $\left(\mathrm{mg} \mathrm{kg}^{-1}\right)$ de carbono potencial de ser liberada no período total de incubação;

$\mathrm{k}$ = constante de velocidade da reação de degradação, em $\operatorname{dia}^{-1}$;

$\mathrm{t}=$ tempo em dias.

$\mathrm{O}$ ajuste ao modelo de cinética química de primeira ordem permitiu obter também a meia-vida de degradação (LATHAM, 1974): $\mathrm{T}_{0,5}=\ln 2 / \mathrm{K}$.

A taxa de degradação foi calculada em função da quantidade de carbono adicionada inicialmente (100\%) e aquela liberada como $\mathrm{C}-\mathrm{CO}_{2}$.

\section{RESULTADOS E DISCUSSÃO}

\section{Solo não contaminado}

As quantidades acumuladas de $\mathrm{C}-\mathrm{CO}_{2}$ liberadas dos tratamentos com quelantes após 50 dias de incubação foram proporcionais às doses, isto é, aumentaram da menor para a maior dose, para ambos os quelantes (Figura 1a). Entretanto, os tratamentos com EDDS exibiram valores 30\% a 60\% maiores em comparação com os tratamentos com EDTA.

O tratamento com glicose, cuja dose de carbono foi semelhante a intermediária dos quelantes, exibiu valor de $\mathrm{C}-\mathrm{CO}_{2}$ acumulado no fim da incubação superior ao tratamento com $500 \mathrm{mg} \mathrm{kg}^{-1}$ de EDTA e inferior à mesma dose, porém de EDDS. Esse comportamento evidencia que o EDDS estimulou mais a atividade microbiana do solo do que a própria glicose, sendo esta última é reconhecidamente de fácil degradação no solo. A liberação de $\mathrm{C}-\mathrm{CO}_{2}$ na menor dose de EDDS foi muito próxima à da

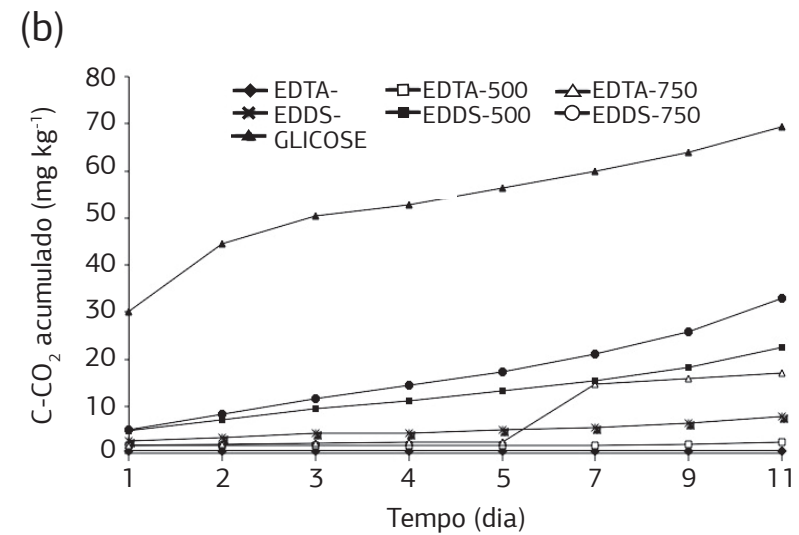

Figura 1. Quantidade de carbono emanado acumulado, na forma de $\mathrm{C}-\mathrm{CO}_{2}$ a partir dos tratamentos EDTA, EDDS e glicose aplicados no solo náo contaminado: (a) Durante todo o período de incubação. (b) Do $1 .^{\circ}$ ao $11 .^{\circ}$ dia de incubaçáo. Os valores descritos na legenda indicam a concentração do quelante $\mathrm{em}_{\mathrm{mg} \mathrm{kg}}{ }^{-1}$ de solo. 
Tabela 1. Valores médios de Carbono adicionado (C adicionado), emanado (C emanado) e mineralizado (C mineralizado) para cada tratamento aplicado no solo não contaminado

\begin{tabular}{|c|c|c|c|c|}
\hline \multirow{2}{*}{ Tratamento } & Dose & C-adicionado & C-emanado & C-mineralizado \\
\hline & \multicolumn{4}{|c|}{$\mathrm{mg} \mathrm{kg}^{-1}$ de solo } \\
\hline Testemunha & 0 & & 320,9 & \\
\hline EDTA & 250 & 80,6 & 312,6 & $-8,3$ \\
\hline EDTA & 500 & 161,2 & 327,9 & 7 \\
\hline EDTA & 750 & 241,7 & 336,4 & 15,5 \\
\hline EDDS & 250 & 80,8 & 413,5 & 92,6 \\
\hline EDDS & 500 & 161,7 & 532,8 & 211,9 \\
\hline EDDS & 750 & 234,3 & 638,2 & 317,4 \\
\hline Glicose & 400 & 159,9 & 429,2 & 108,3 \\
\hline
\end{tabular}

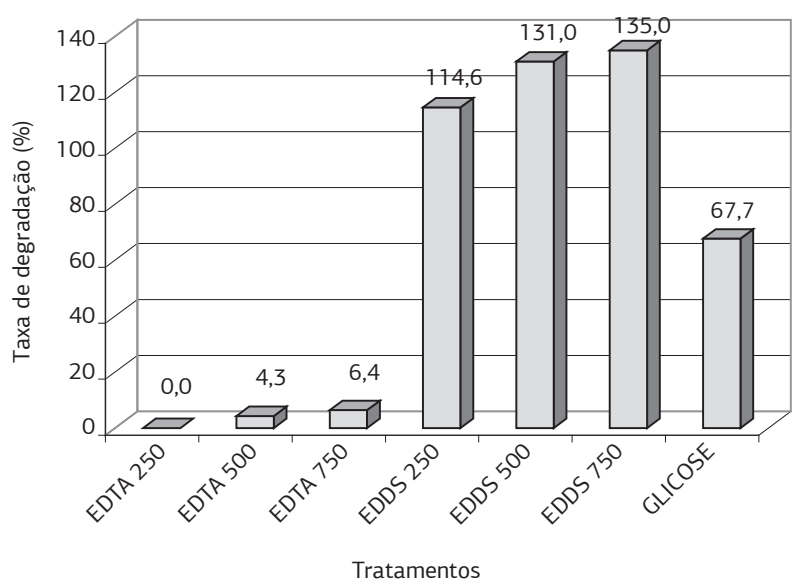

Figura 2. Taxa de degradação no fim da incubação para os tratamentos no solo náo contaminado. Os valores descritos no eixo $\mathrm{x}$ indicam a concentração dos tratamentos em $\mathrm{mg} \mathrm{kg}^{-1}$ de solo.

glicose, mas a quantidade de carbono adicionada nesta última foi praticamente o dobro, o que confirma o estímulo à atividade microbiana proporcionado pelo EDDS.

Um aspecto interessante observado foi a ocorrência, para todos os tratamentos, com exceção da glicose, da fase lag, que é uma fase de adaptação da comunidade microbiana às novas condiçôes do meio (Figura 1a, b). Essa fase duração variável, mas ficou entre os 15 e 20 primeiros dias de incubação e pode ser mais bem observada na figura $1 \mathrm{~b}$. Os acréscimos de C- $\mathrm{CO}_{2}$ ocorreram efetivamente nos tratamentos com EDTA após o $5 .^{\circ}, 14 .^{\circ}$ e $32 .^{\circ}$ dias de incubação das doses de 750, 500 e $250 \mathrm{mg} \mathrm{kg}^{-1}$ respectivamente.

O EDDS foi totalmente degradado em 50 dias de incubação, independentemente da dose aplicada, enquanto o EDTA exibiu valor médio da ordem de $8 \%$ e $12 \%$ de degradação, nas doses de 500 e $750 \mathrm{mg} \mathrm{kg}^{-1}$, no mesmo período (Tabela 1). Os valores de taxa de degradação para os quelantes e glicose são apresentados na figura 2. Os baixos valores das taxas de degradação com a aplicação das doses de EDTA no solo são concordantes com dados da literatura quanto à elevada persistência dessa espécie química no solo (Grŏman et al., 2003). Apesar disso, so- mente na menor dose do quelante houve algum tipo de prejuízo à atividade microbiana do solo, e nas outras duas doses testadas observou-se aumento da atividade microbiana em comparaçáo com o controle (Tabela 1).

A partir dos valores de carbono degradado no período de incubação foram testados ajustes ao modelo de cinética de primeira ordem, mas não houve ajuste satisfatório para os tratamentos com EDTA nas duas menores doses e em todas as doses de EDDS. Nas doses de 250 e $500 \mathrm{mg} \mathrm{kg}^{-1}$ de EDTA verificou-se que os valores de $\mathrm{C}-\mathrm{CO}_{2}$ liberados foram baixos e praticamente constantes até o $38 .^{\circ}$ e $47 .^{\circ}$ dias de incubação. $\mathrm{O}$ aumento ocorrido no período subsequente não permitiu o ajuste ao modelo de cinética em questão. Nos tratamentos com EDDS o motivo do ajuste insatisfatório ao modelo proposto foi a predominância de aumento exponencial, sem estabilização, após o $24 .^{\circ}$ dia até o fim do período avaliado. Considerando que $100 \%$ do EDDS foi degradado no período considerado e que não houve estabilizaçáo do $\mathrm{C}-\mathrm{CO}_{2}$ liberado, conclui-se que o quelante estimulou a degradação do carbono original do solo, efeito este conhecido como "efeito priming".

A causa provável do não ajuste da cinética química pode ser explicada pela ocorrência da fase lag e efeito priming. A adição do quelante EDTA resultaria em um efeito priming negativo. Este tipo de efeito, de acordo com a classificação de efeitos priming organizada por KuzYaKov et al. (2000), ocorreria pela adição de substâncias tóxicas, que por sua vez, causariam a inibição direta da atividade de microrganismos ou de suas enzimas. Esse fato pode ser confirmado por GRŎMAN et al. (2003), que estudaram a toxicidade do EDTA em relação ao EDDS por meio da técnica de análise de ácidos graxos dos fosfolipídeos (PLFA) que possibilitou também a comparação da gordura trans em relação à cis dos PFLAs, verificando-se o aumento da primeira, associada à condiçáo de inaniçáo ou estresse dos microrganismos em ambientes naturais, maior na presença do EDTA em relação ao EDDS.

Com relação à adição do EDDS, haveria a ocorrência do efeito priming positivo que, de acordo com Kuzyakov et al. (2000), o mecanismo do efeito ocorreria pela adição 
de substâncias orgânicas facilmente degradáveis que causariam aumento na atividade dos microrganismos e aceleração da mineralização da matéria orgânica originalmente presente no solo. No ensaio de GrŏmAn et al. (2003), a adição do EDDS foi menos tóxica aos fungos do que a adição do EDTA e causou menos estresse aos microrganismos do solo. O EDDS é uma substância de ocorrência natural e esta é a razão para sua baixa toxicidade. Foi primeiro isolado de um filtrado de cultura do actinomiceto Amycolatopsis orientalis e detectado em uma varredura de seleção de antibiótico.

$\mathrm{Na}$ maior dose de EDTA o ajuste teve valor de $\mathrm{r}$ igual a 0,955 e os parâmetros da equação de cinética foram: $\mathrm{C}_{0}=26,912 \mathrm{mg} \mathrm{kg}^{-1}$ e $\mathrm{k}=0,066 \mathrm{dia}^{-1}$. O tratamento glicose também exibiu elevado valor de $\mathrm{r}(0,901)$ e os parâmetros da equação de cinética foram: $\mathrm{C}_{0}=95,260 \mathrm{mg} \mathrm{kg}^{-1}$ e $\mathrm{k}=0,161 \mathrm{dia}^{-1}$.

Comparando-se a velocidade de degradação da glicose em relação ao EDTA, percebe-se que foi 2,5 vezes maior que a do EDTA na dose de $750 \mathrm{mg} \mathrm{kg}^{-1}$, responsável pela maior velocidade de degradação do quelante, por isso os valores de $T_{0,5}$ também foram distintos, 0,7 e 10,4 dias respectivamente. Esse processo significa que no tratamento com glicose, metade do total de $\mathrm{C}-\mathrm{CO}_{2}$ liberado, no período total de avaliaçáo, ocorreu em menos de um dia, enquanto na dose de $750 \mathrm{mg} \mathrm{kg}^{-1}$ de EDTA o tempo para liberação de $50 \%$ do total de $\mathrm{C}-\mathrm{CO}_{2}$ foi de 10 dias.

\section{Solo contaminado}

A liberação de $\mathrm{C}-\mathrm{CO}_{2}$ nos tratamentos com EDDS superou a verificada nos tratamentos com EDTA, bem como também a determinada no tratamento com glicose (Figura 3), semelhante ao ocorrido no solo não contaminado. Náo houve incremento proporcional do $\mathrm{C}-\mathrm{CO}_{2}$ acu- mulado no fim de 43 dias de incubação em função do aumento da dose dos quelantes, a dose de $750 \mathrm{mg} \mathrm{kg}^{-1}$ proporcionou a maior liberaçáo de $\mathrm{C}-\mathrm{CO}_{2}$ para ambos os quelantes, seguido da dose de $250 \mathrm{mg} \mathrm{kg}^{-1}$ e por último a de $500 \mathrm{mg} \mathrm{kg}^{-1}$.

A degradação dos quelantes diminuiu com o aumento das doses aplicadas, variando entre $50 \%$ a $19 \%$ para o EDTA e entre $211 \%$ e $67 \%$ para o EDDS (Figura 4). Novamente o EDDS foi mais degradável após aplicação no solo, mesmo com o EDTA exibindo valores de degradação superiores àqueles observados no solo não contaminado. De modo geral, a maior degradação do EDTA neste solo (inferior a 10 dias em todos os tratamentos) deve-se pela não-ocorrência da fase de adaptação da comunidade microbiana (fase lag). Essas e outras diferenças devem ser inerentes às características originais, como teor de matéria orgânica, concentraçóes de nutrientes e pH. O importante é que em ambos, o EDDS foi mais degradável que o EDTA, o que significa que o EDDS possui um menor tempo ativo no solo e esse fato deve nortear as técnicas de manejo de sua aplicação, uma vez que deve estar atuando sincronizadamente com o período de maior absorção pela plantas.

O EDDS na dose de $500 \mathrm{mg} \mathrm{kg}^{-1}$ proporcionou maior degradação que a glicose, lembrando que a quantidade de carbono adicionado por esses tratamentos foi semelhante (Tabela 2 e Figura 4). A taxa de degradaçáo da glicose foi semelhante nos dois solos, em contraposição ao verificado nos tratamentos com quelantes, o que deve estar relacionado ao maior potencial de liberaçáo de $\mathrm{C}-\mathrm{CO}_{2}$ no solo contaminado, função da alta concentração de carbono ( 5,6 vezes maior que no solo não contaminado), aliado ao efeito de melhoria das condiçóes edáficas proporcionada pelo EDTA e EDDS. Esse fato significa que provavelmente parte do carbono liberado dos tratamentos com quelante seja proveniente da degradação do carbono original do

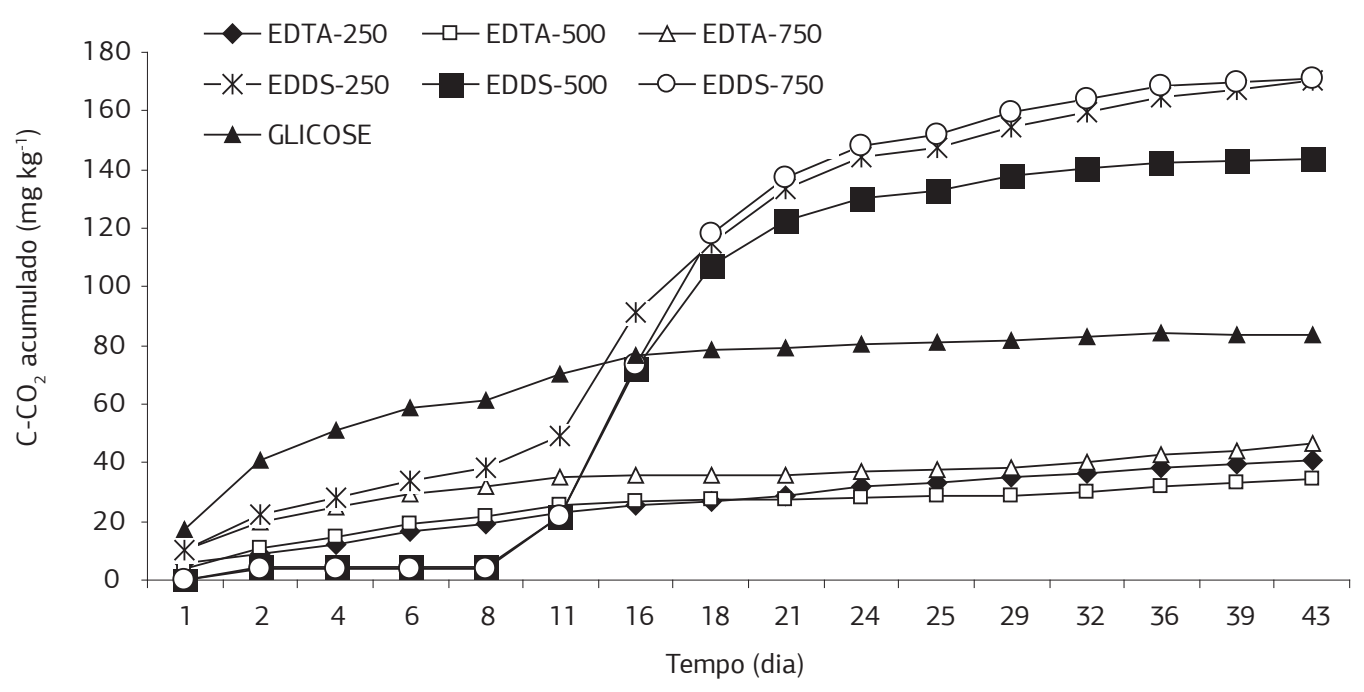

Figura 3. Quantidade de carbono emanado acumulado, na forma de $\mathrm{C}-\mathrm{CO}_{2}$ a partir dos tratamentos EDTA, EDDS e glicose aplicados no solo contaminado. Os valores descritos na legenda indicam a concentraçáo do quelante em $\mathrm{mg} \mathrm{kg}^{-1} \mathrm{de}$ solo. 
solo (efeito priming), no caso da menor dose $250 \mathrm{mg} \mathrm{kg}^{-1}$ de EDDS (Tabela 2).

Os metais pesados podem prejudicar a comunidade microbiana do solo (InSAM et al., 1996; BRookes e Macgrath, 1984; Fliebbach et al., 1994; Aceves et al., 1999), no entanto, a aplicação dos quelantes EDTA e EDDS, com base nos valores das taxas de degradação analisados neste trabalho, podem ter amenizado estes efeitos.

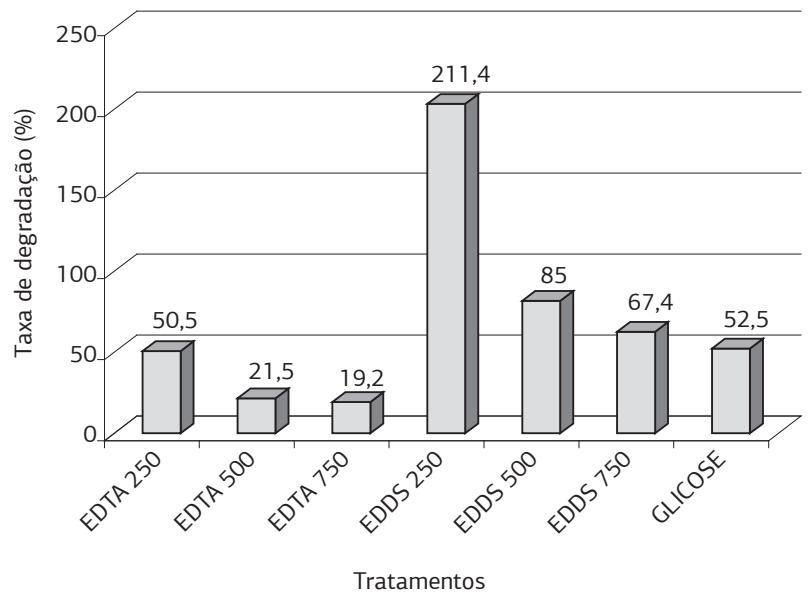

Figura 4. Taxa de degradação no fimda incubação para os tratamentos no solo contaminado. Os valores descritos no eixo $\mathrm{x}$ indicam a concentração dos tratamentos em $\mathrm{mg} \mathrm{kg}^{-1}$ de solo.
Todos os tratamentos tiveram bom ajuste dos dados de $\mathrm{C}-\mathrm{CO}_{2}$ ao modelo de cinética química de primeira ordem, como se pode observar por meio dos valores de $\mathrm{r}$ da tabela 3. No caso dos tratamentos com EDDS, os primeiros 15 dias de avaliação náo foram utilizados para o ajuste, uma vez que correspondeu ao tempo da fase lag nesses tratamentos. Tal artifício também foi testado no caso do solo não contaminado, porém, mesmo assim, os ajustes não foram satisfatórios.

A velocidade de degradação do EDTA, avaliada por meio dos valores de $\mathrm{k}$ (Tabela 3 ), foram proporcionais ao aumento da dose, ou seja, dobraram da dose mais baixa (250 mg kg ${ }^{-1}$ ) para a intermediária e aumentaram 50\% da dose intermediária para a mais alta. Esse tipo de relação evidencia a dependência da degradação em função da dose, o que não ocorreu nos tratamentos com EDDS, significando que este último quelante é menos impactante na microbiota do solo comparativamente ao EDTA (GrŏMAN et al., 2003)

Os valores de C- $\mathrm{CO}_{2}$ também podem ser utilizados para calcular a taxa de degradaçáo de compostos orgânicos após aplicação no solo, com a vantagem de se eliminar algumas distorçóes em função da variação dos dados originais, desde que o ajuste tenha sido adequado (elevados valores de r). Usando os valores de $\mathrm{C}_{0}$

Tabela 2. Valores médios de Carbono adicionado (C adicionado), emanado (C emanado) e mineralizado (C mineralizado) para cada tratamento aplicado no solo contaminado

\begin{tabular}{|c|c|c|c|c|}
\hline \multirow{2}{*}{ Tratamento } & Dose & C-adicionado & C-emanado & C-mineralizado \\
\hline & \multicolumn{4}{|c|}{$\mathrm{mg} \mathrm{kg}^{-1}$ de solo } \\
\hline Testemunha & 0 & & 627,4 & \\
\hline EDTA & 250 & 80,6 & 668,1 & 40,7 \\
\hline EDTA & 500 & 161,2 & 662,1 & 34,7 \\
\hline EDTA & 750 & 241,7 & 673,9 & 46,5 \\
\hline EDDS & 250 & 80,8 & 798,2 & 170,8 \\
\hline EDDS & 500 & 161,7 & 764,8 & 137,4 \\
\hline EDDS & 750 & 234,3 & 785,3 & 157,9 \\
\hline Glicose & 400 & 159,9 & 711,4 & 83,7 \\
\hline
\end{tabular}

Tabela 3. Parâmetros de cinética química e meia-vida de degradação $\left(\mathrm{T} 0,5^{\mathrm{d}}\right)$ obtidos a partir da tentativa de ajuste dos dados de C-degradado a equaçóes de cinética química de primeira ordem durante o período de incubação para o solo contaminado. $\mathrm{C}_{\text {degradado= }} \mathrm{C}_{0}$. $\left(1-\mathrm{e}^{-\mathrm{k} . t}\right) \mathrm{b}$

\begin{tabular}{|c|c|c|c|c|c|}
\hline \multirow[t]{2}{*}{ Tratamento } & Dose & $C_{0}$ & k & $T_{0,5}{ }^{d}$ & \multirow[t]{2}{*}{$\mathbf{r}$} \\
\hline & \multicolumn{2}{|c|}{$\mathrm{mg} \mathrm{kg}^{-1}$} & dia $^{-1}$ & dias & \\
\hline EDTA & 250 & 40,425 & 0,072 & 9,6 & 0,983 \\
\hline EDTA & 500 & 30,709 & 0,153 & 4,5 & 0,977 \\
\hline EDTA & 750 & 39,446 & 0,244 & 2,8 & 0,941 \\
\hline EDDS & 250 & 200,251 & 0,048 & 14,3 & 0,961 \\
\hline EDDS & 500 & 165,801 & 0,056 & 12,5 & 0,998 \\
\hline EDDS & 750 & 212,720 & 0,044 & 15,9 & 0,898 \\
\hline Glicose & 400 & 81,101 & 0,232 & 3,0 & 0,977 \\
\hline
\end{tabular}

C-degradado = quantidade de carbono $\left(\mathrm{mg} \mathrm{kg}^{-1}\right)$ emanado na forma de $\mathrm{CO}_{2}$ no tempo $\mathrm{t} ; \mathrm{C}_{0}=$ carbono $\left(\mathrm{mg} \mathrm{kg}^{-1}\right)$ potencialmente mineralizável em 43 dias de incubaçáo; $\mathrm{k}=$ constante de velocidade da reaçáo de degradaçáo do carbono orgânico do quelante $\left(\mathrm{dia}^{-1}\right) ; \mathrm{T}=$ tempo de incubaçáo em dias; $\mathrm{r}=$ coeficiente de correlaçáo. 
da tabela 3, têm-se taxas de degradação iguais a 50,3\%; $19,1 \%$ e $16,3 \%$ para as doses 250,500 e $750 \mathrm{mg} \mathrm{kg}^{-1}$ de EDTA e iguais a $247,8 \% ; 102,5 \%$; e $90,8 \%$ para as doses 250, 500 e $750 \mathrm{mg} \mathrm{kg}^{-1}$ de EDDS. Analisando esses resultados, percebe-se que o EDDS foi totalmente degradado no período de 43 dias de incubação, enquanto o EDTA degradou apenas $30 \%$, o que implica em cessaçáo do efeito do EDDS como quelante e continuidade da açấo do EDTA no solo após esse período.

Os valores de $T_{0,5}$ do EDDS ficaram entre 12 e 16 dias, uma vez que acompanham os valores de velocidade de degradação $(\mathrm{k})$, e permitem inferir que após cerca de 15 dias, o efeito do quelante do EDDS deve ser reduzido à metade, pois ele foi totalmente degradado no período de incubação. Os valores de meia-vida, um pouco mais altos do que aqueles constatados em literatura, pode ser causado pela presença de metais pesados, entre eles Zn e $\mathrm{Cu}$. Segundo Vandevivere et al. (2001), além do EDDS não complexado, os complexos metálicos com EDDS são prontamente biodegradáveis. Os complexos formados com $\mathrm{Ca}, \mathrm{Mg}, \mathrm{Cd}, \mathrm{Fe}(\mathrm{III}), \mathrm{Al}, \mathrm{Pb}$ e $\mathrm{Cr}$ (III) foram facilmente degradados pelos microrganismos em sistemas de lodo ativados, enquanto a degradação do complexo EDDS-Zn somente ocorreu depois de uma extensiva fase lag. $\mathrm{O}$ complexo $\mathrm{Cu}-\mathrm{EDDS}$ permaneceu não degradado. Dessa forma, a presença dos metais pesados $\mathrm{Zn}$ e Cu foram os prováveis responsáveis pela fase lag observada.

Para o EDTA deve-se ter mais cuidado ao analisar os resultados, pois os valores médios de degradação para as três doses foi de $29 \%$ e a meia vida foi de seis dias, significando que após esse período, o efeito quelante devido à degradação do EDTA foi reduzido em apenas 15\%.

\section{CONCLUSÃO}

O agente quelante EDDS foi mais facilmente degradável tanto no Cambissolo háplico contaminado quanto no Latossolo Vermelho-Amarelo sem contaminação por metais pesados, comparativamente ao EDTA. No solo a ser remediado, o EDDS foi totalmente degrado em até 50 dias após aplicação no solo, com sua atividade reduzida em $50 \%$ após os primeiros 15 dias, enquanto o EDTA foi mais persistente no ambiente, podendo em 50 dias ter seu efeito quelante reduzido em apenas 15\% devido à baixa degradação da molécula.

A aplicação de EDDS como prática remediadora de solos contaminados por metais pesados deve considerar a rápida degradação dessa molécula e, desde que com eficiência aceitável, deve ser preferida ao EDTA, uma vez que incorre em menores riscos de efeitos adversos em função da persistência da molécula no ambiente.

\section{AGRADECIMENTOS}

À Fundação Instituto de Terras do Estado de São Paulo, pela liberaçáo, por meio do Programa de Incentivo à Educação Formal e Especialização Profissional, e às técnicas de laboratório Luciana Damasceno de Souza e Luciana Taminato Imazaki, pela ajuda preciosa nos experimentos. Ao CNPq pelo auxílio financeiro ao desenvolvimento do projeto.

\section{REFERÊNCIAS}

ACEVES, M.B.; GRACE, C.; ANSORENA, J.; DENDOOVEN, L.; BROOKES, P.C. Soil microbial biomass and organic $\mathrm{C}$ in a gradient of zinc concentration in soils around a mine spoil tip. Soil Biology and Biochemistry, v.31, p.867-876, 1999.

BROOKES, P.C.; MACGRATH, S.P. Effects of metal toxicity on the size of the soil microbial biomass. Journal of Soil Science, v.35, p.341-346, 1984.

COSCIONE, A.R.; ANDRADE, C.A. Protocolos para a avaliação da dinâmica de resíduos orgânicos no solo. In Análise química de resíduos sólidos para monitoramento e estudos agroambientais. Campinas: Instituto Agronômico, 2006. p.159-177.

CURVE EXPERT 1.3. Disponível em: http://www.ebicom. net/ dhyams/cftp.htm Acesso em: 10 de março de 2007.

EMBRAPA. Centro Nacional de Pesquisa de Solos. Sistema Brasileiro de Classificação de Solos. Brasília, 1999. 412p.

FLIEBBACH, A.; MARTENS, A.; REBER, H.H. Soil microbial biomass and microbial activity in soils treated with heavy metal contaminated sewage-sludge. Soil Biology and Biochesmistry, v.26, p.1201-1205, 1994.

GRŎMAN, H.; VODNIK, D.; VELIKONJA-BOLTA, S.; LEŠTAN, D. Ethylenediaminedisuccinate as a new chelate for environmentally safe enhanced lead phytoextraction, Environmental Science Technology, v.32, p.500-506, 2003.

INSAM, H.; HUTCHINSON, T. C.; REBER, H. H. Effects of heavy metal stress on the metabolic quotient of the soil microflora. Soil Biology and Biochemistry, v.28, p. 691-694, 1996.

JAWORSKA, J.S.; SCHOWANEK, D.; FEIJTEL, T.C.J. Environmental risk assessment for trisodeium $[S, S]$ etylene diamine disuccinate, a biodegradable chelator used in detergent applications. v.38, p.3597-3625, 1999.

KUZYAKOV, Y.; FRIEDEL, J.K.; STAHR, K. Review of mechanisms and quantification of priming effects. Soil Biology and Biochemistry, v.32, p.1485-1498, 2000.

LASAT, M.M. Phytoextraction of toxic metals: a review of biological mechanisms. Journal of Environmental Quality, v.31, p.109-120, 2002. 
LATHAM, J.L. Cinética elementar de reação. São Paulo: E. Blücher, 1974. 112p.

MADRID, F.; LIPHADZI, M.S.; KIRKHAM, M.B. Heavy-metal displacement in chelate-irrigated soil during phytoremediation. Journal of Hydrology, v.272, p.107-119, 2003.

OLIVEIRA, J.B.; LIER, Q.; NIESTEN, B.; MENK, J.R.F. Levantamento pedológico detalhado do Centro Experimental de Campinas. Campinas: Instituto Agronômico, 2007. (Boletim Técnico, submetido para publicação).
RAIJ, B. VAN; ANDRADE, J.C.; CANTARELLA, H.; QUAGGIO, J.A. Análise química para avaliação da fertilidade de solos tropicais. Campinas: Instituto Agronômico, 2001. 285p.

RODELLA, A.A.; SABOYA, L.V. Calibration for condutimetric determination of carbon dioxide. Soil Biology and Biochemistry, v.31, p.2059-2060, 1999.

VANDEVIVERE, P.; SAVEYN, H.; VERSTRAETRE, W.; FEIJTEL, T.C.J.; SCHOWANEK, D.R. Biodegradation of metal [S,S] - EDDS Complexes. Environmental Science and Technology, v.35, p.1765-1770, 2001. 